\title{
Sorgfaltskriterien im Umgang mit Suizidbeihilfe
}

\author{
Nationale Ethikkommission \\ im Bereich Humanmedizin \\ (NEK)
}

Korrespondenz:

Dr. iur. Sibylle Schürch

Nationale Ethikkommission

Bereich Humanmedizin

Bundesamt für Gesundheit

Seilerstrasse 8

CH-3003 Bern

Tel. 0313249365

Fax 0313226233

sibylle.schuerch@bag.admin.ch

www.nek-cne.ch

\section{Einleitung}

In ihrer Stellungnahme Nr. 9/2005 [1] hat die Nationale Ethikkommission dem Gesetzgeber unter anderem empfohlen, Organisationen, die in der Schweiz im Schutz von Art. 115 StGB Beihilfe zum Suizid anbieten und durchführen, einer staatlichen Aufsicht zu unterstellen. Rechtliche Vorgaben sollen sicherstellen, dass in der Anwendung von Art. 115 StGB neben dem Respekt vor der Selbstbestimmung auch die Fürsorge für suizidgefährdete Menschen im Sinn des Schutzes ihres Lebens gleichgewichtige Berücksichtigung findet.

Konkret sollen nach Auffassung der Ethikkommission eine Reihe von minimalen Sorgfaltskriterien für die Praxis der organisierten Suizidbeihilfe vorgeschrieben werden. In der Stellungnahme Nr. 9/2005 ist aber - abgesehen von einigen richtungsweisenden Hinweisen offengeblieben, worin die in einer Aufsichtsregelung zu verlangenden Kriterien bestehen sollen. Mit dem vorliegenden Papier füllt die Kommission diese Lücke. Die hier vorgelegten Empfehlungen richten sich auch an die Praxis.

Die darin formulierten Forderungen verstehen sich nicht als Kriterien, die, wenn sie eingehalten werden, $\mathrm{zu}$ einer staatlichen oder gesellschaftlichen Anerkennung von Suizidhilfeorganisationen oder ihrer Praxis im Einzelfall führen können. Die Kriterien verstehen sich vielmehr als notwendige Minimalstandards, die keine Verantwortung von den Organisationen wegnehmen. Es ist die Meinung der Kommission, dass die weitgehende Freiheit für das organisierte Angebot von Suizidbeihilfe, die vom schweizerischen Strafrecht in Form des geltenden Art. 115 StGB geschaffen ist, auch eine gesellschaftliche Verantwortung zum Schutz der Betroffenen nach sich zieht. Die vorliegende Empfehlung ist von diesem Anliegen des Schutzes der Betroffenen getragen.

Die Empfehlungen wollen keine zeitlose Gültigkeit beanspruchen. Nach Vorliegen von Erfahrungen aus der Praxis sollen sie vielmehr neu diskutiert und gegebenenfalls revidiert werden können.
Der Diskussion und Formulierung dieser Empfehlungen in der Kommission ist eine Anhörung von Vertreterinnen und Vertretern von drei Suizidhilfeorganisationen, der Rechtsmedizin, eines kantonsärztlichen Dienstes und einer kantonalen Staatsanwaltschaft vorausgegangen. Hintergrund ist auch die breitgeführte Diskussion im Vorfeld der Stellungnahme 9/2005, die im Buch «Beihilfe zum Suizid in der Schweiz. Beiträge aus Ethik, Recht und Medizin» [2] ihren Niederschlag gefunden hat.

Die zwölf Empfehlungen in der Stellungnahme 9/2005 «Beihilfe zum Suizid» bilden einen integralen Bestandteil dieser Empfehlungen.

\section{Ziel und Hintergrund}

Es ist das Ziel dieser Empfehlungen darzustellen, welchen Schutz Personen, die den Wunsch haben, zu sterben, gegenüber dem organisierten Angebot einer Hilfe zur Selbsttötung brauchen. Dies betrifft auch sterbewillige Menschen aus dem Ausland.

Im Hintergrund steht die strafrechtliche Regelung gemäss Art. 115 StGB, die Beihilfe zur Selbsttötung ermöglicht, soweit sie nicht «aus selbstsüchtigen Beweggründen» erfolgt. Es gibt in der Schweiz heute keine weitergehenden gesetzlichen Anforderungen, die beispielsweise sicherstellen, dass der Beihilfe zum Suizid eine genügend sorgfältige, auch Alternativoptionen einschliessende Abklärung vorausgeht.

Aus ethischer Sicht bewegt sich die Suizidbeihilfe zwischen zwei Polen: einerseits der gebotenen Fürsorge für suizidgefährdete Menschen, andererseits dem Respekt vor der Selbstbestimmung des Suizidwilligen. Beide Pole sind gleichermassen zu berücksichtigen. Die Kommission stützt aus ethischen Beweggründen die in der Schweiz gegebene Freiheit, Beihilfe zur Selbsttötung zu leisten. Die Empfehlung 5 (in 9/2005) stellte in bezug auf die Tätigkeit von Sterbehilfeorganisationen aber einen zusätzlichen gesetzlichen Regelungsbedarf fest. Die Kommission hatte zum Inhalt dieser Kriterien zwar bereits einige Kernpunkte festgehalten, je- 
doch keine Liste von Kriterien erarbeitet. Diese Lücke möchte sie nun füllen und gleichzeitig auf Missbrauchsgefahren aufmerksam machen.

Die liberale rechtliche Situation lässt es zu, dass sich Sterbehilfeorganisationen innerhalb des rechtlichen Rahmens frei organisieren, dass sie sich selber Richtlinien geben und ihre Tätigkeit ausüben können. Zwischen der Hilfeleistung innerhalb einer Familien- oder Freundesbeziehung und dem organisierten Angebot zu einem sicheren und schmerzfreien Tod besteht ein wesentlicher Unterschied. Die Tatsache eines organisierten Angebotes verändert die Situation für Menschen mit einem Suizidwunsch. Es besteht die Gefahr, dass diese Organisationen einseitig auf das Prinzip der Selbstbestimmung des Menschen abstellen und dabei dem Schutz des Lebens, dem Gebot der Fürsorge im Sinn der Verantwortung für suizidgefährdete Menschen zuwenig Beachtung schenken.

Für die Beteiligung von Ärztinnen und Ärzten an assistierten Suiziden im Kontext der Betreuung von Patienten und Patientinnen am Lebensende hat die Schweizerische Akademie der Medizinischen Wissenschaften (SAMW) im Jahr 2004 neue Richtlinien erlassen [3]. Der Bundesrat hat es mit Entscheid vom 31. Mai 2006 zwar abgelehnt, eine staatliche Aufsicht über Sterbehilfeorganisationen einzurichten. In seinem Bericht anerkennt er gleichwohl, dass es Missbrauchspotentiale gibt, insbesondere bei schutzbedürftigen Personengruppen wie Jugendlichen, psychisch- und terminalkranken Personen [4]. Auf rechtlicher Ebene sind die Behörden in der Pflicht, Missbrauch aufzudecken und strafrechtlich zu untersuchen. Diese Arbeit kann durch ethische Richtlinien unterstützt werden, welche die im Einzelfall aus Sicht des Lebensschutzes ausschlaggebenden Aspekte der Urteilsund Handlungsfähigkeit der Suizidwilligen, der Abklärungspflicht im Einzelfall und die Vertretungsverhältnisse differenzierter darstellen.

\section{Definition der organisierten Suizidbeihilfe}

Als «organisierte Suizidbeihilfe» bzw. als «Suizidhilfeorganisation» soll im Rahmen dieser Empfehlungen eine Tätigkeit verstanden werden, die darin besteht, Hilfeleistungen für die Selbsttötung an vorher Unbekannte anzubieten oder Unbekannten zur Verfügung zu stellen.

Diese Tätigkeit kann von Vereinen oder ähnlich organisierten Körperschaften oder von Einzelpersonen (wenn sie die Hilfe zur Selbsttötung regelmässig durchführen und/oder sie an Unbekannte zur Verfügung stellen) angeboten werden. Eine Hilfeleistung in einer einzelnen, engen persönlichen oder familiären Beziehung sowie eine einzelne und einzigartige Hilfeleistung im Rahmen einer umfassenden Beziehung zwischen Ärztin/Arzt und Patientin/Patient steht hingegen für diese Empfehlungen nicht im Zentrum, ebensowenig der Suizid als solcher.

\section{Empfehlungen bezüglich Abklärung von suizidwilligen Personen}

Folgende Mindestanforderungen müssen überprüft, erfüllt und dokumentiert sein, damit aus ethischer Sicht Suizidbeihilfe geleistet werden darf.

\section{Es besteht Urteilsfähigkeit im Hinblick auf die Entscheidung, das eigene Leben mit Hilfe eines Dritten zu beenden}

Die Urteilsfähigkeit kann nur in persönlichen, länger dauernden und wiederholten Gesprächen abgeklärt werden. Deren minimale Zeitdauer soll sich in erster Linie nach den konkreten Umständen richten, die die Lebenssituation der sterbewilligen Person charakterisieren (ihre Bedürfnisse, die Komplexität der Probleme, die zum Suizidwunsch führen, der Krankheitsverlauf usw.). Sie soll nicht durch die Einschränkungen auf der Seite der Sterbehelfer bestimmt sein (z. B. durch ihre zeitliche Verfügbarkeit oder ihre Distanz zum Lebensort des Sterbewilligen).

Die erwachsene Person im Besitz der Urteilsfähigkeit ist im allgemeinen die beste Zeugin und die beste Richterin ihrer Situation. Sie kann beurteilen, ob ihr Leiden zu gross ist. Wichtig ist, dass die eigene subjektive Sicht der suizidwilligen Person den Ausschlag gibt und nicht eine Beurteilung nach fremden Kriterien.

Solange Zweifel an der Urteilsfähigkeit bestehen, darf Suizidbeihilfe nicht geleistet werden.

\section{Der Suizidwunsch ist aus einem schweren, krankheitsbedingten Leiden entstanden}

Unter dem Aspekt des Schutzes des Lebens erscheint es als ethisch fragwürdig, organisierte Suizidbeihilfe an Personen zu leisten, die mit ihrem Leben nicht zufrieden sind, aus philosophischer Überzeugung nicht an ihrem Leben hängen oder eine lebensverneinende Haltung haben. Autonomie ist ein zentraler Wert, aber für die organisierte Suizidbeihilfe nicht der einzige. Der Schutz des Lebens und sozialethische Gründe bilden in nichtkrankheitsbedingten Fällen eine Grenze. Wenn kein von der Deklaration des Willens unabhängiger, darstellbarer Grund vorliegt, muss für die Organisation der Aspekt der Fürsorge (im Sinn des Lebensschutzes) Vorrang haben. Es sollen darum nur Personen in Frage kommen, die krankheitsbedingt* schwer leiden. 
Psychischkranken Menschen, bei denen die Suizidalität ein Ausdruck oder Symptom der Erkrankung ist, soll keine Suizidbeihilfe gewährt werden

Psychischkranke möchten sich oft aus einem vorübergehenden oder behandelbaren Leiden das Leben nehmen. Zur Beurteilung, ob eine psychische Krankheit vorliegt, sind adäquate Kenntnisse psychischer Krankheiten erforderlich. Im Zweifelsfall ist der Beizug einer Fachperson nötig.

\section{Der Sterbewunsch ist dauerhaft} und konstant. Er ist nicht aus einem Affekt oder aus einer absehbar vorübergehenden Krise entstanden

Der Faktor Zeit kann die Lebenslage, in der ein Sterbewunsch entsteht, verändern. Zudem ist die Endgültigkeit des Sterbewunsches davon abhängig, dass er im Hinblick auf die Gesamtsituation genügend reflektiert ist. Dies bedarf ausreichender Zeit («Bedenkzeit»). Es ist allerdings nicht möglich, ein objektives Zeitmass anzugeben, bis ein Sterbewunsch als «konstant» gelten kann. Wieviel Zeit eingeräumt werden muss, soll einerseits davon abhängen, ob sich die Lebenslage aus Sicht der abklärenden Person absehbar wesentlich verändern und sich damit der Sterbewunsch verringern kann. Andererseits soll in die Beurteilung der nötigen Zeitspanne auch einfliessen, ob die Gesamtsituation genügend bedacht werden konnte.

\section{Der Wunsch zum Suizid ist frei von äusserem} Druck zustande gekommen

Beispiele äusseren Druckes können sein: Druck von seiten Angehöriger, soziale Isolation, den Angehörigen «zur Last zu fallen», finanzielle Engpässe, die bei der betroffenen Person auch Angst vor fehlender oder mangelhafter Betreuung und Fürsorge entstehen lassen. Solche beispielhaft genannten Druckfaktoren dürfen für den Sterbewunsch nicht ausschlaggebend sein.

Wichtig ist, dabei im Auge zu behalten, dass Druckfaktoren auch als subjektive Befürchtungen vorhanden sein können, ohne objektiv feststellbar zu sein. Diese Faktoren entfalten dennoch ihre Wirkung.

Die Abklärung, ob die Entscheidung frei von äusserem Druck zustande gekommen ist, verlangt zwingend das individuelle Gespräch, ohne Beisein von Angehörigen oder Dritten, von denen eine mögliche Beeinflussung ausgehen könnte. Dies schliesst eine gemeinsame Abklärung von zwei oder mehreren Personen aus, die gemeinsam Suizid begehen möchten (z. B. Paarsuizide). Denn dort ist das Risiko hoch, dass die
Initiative nicht von beiden Partnern gleichermassen ausgeht und der Entschluss für den einen der Partner nicht frei zustande kommt.

Alle alternativen Optionen sind abgeklärt, mit dem Suizidwilligen erwogen und geprüft sowie gemäss seinem Wunsch ausgeschöpft Die Situation muss daraufhin geprüft werden, ob sie auf anderem Weg zugunsten der Person verändert werden kann. Bei der Frage, wie weit man gehen muss, um alternative Optionen (wie ärztliche Behandlung, Sozialhilfe, Therapie) nicht nur abzuklären, sondern auch auszuschöpfen, muss der Wunsch der sterbewilligen Person berücksichtigt werden.

Persönliche, mehrmalige Kontakte und intensive Gespräche sind unabdingbar.

Eine Abklärung aufgrund einer einmaligen Begegnung oder auf dem Korrespondenzweg ist ausgeschlossen

Die Feststellung der Urteilsfähigkeit bleibt auch bei sorgfältigster Abklärung von der subjektiven Wahrnehmung, den Werten, der Lebenserfahrung und der Gesprächsfähigkeit des Abklärenden abhängig. Entsprechend hoch sind die Anforderungen an die Person, die die Abklärungen durchführt.

Es ist wesentlich, dass die Lebenssituation einer suizidwilligen Person erfasst und dokumentiert wird. Dazu gehören die Kenntnisse über das schwere, krankheitsbedingte Leiden und Informationen über das psychosoziale Umfeld sowie die Lebensgeschichte, unter Berücksichtigung des Rechtes des Suizidwilligen auf Achtung der Privatsphäre. Dazu sind mehrmalige und persönliche Begegnungen und Gespräche unabdingbar. So kann garantiert werden, dass die Konstanz des Sterbewunsches über längere Zeit überprüft und bestätigt wird. Gleichzeitig ist aber darauf zu achten, dass die sorgfältige Abklärung die Leidenszeit nicht unnötig verlängert.

\section{Eine unabhängige Zweitmeinung kommt zum gleichen Schluss}

Es ist wichtig, dass die Beurteilung der Situation nicht nur durch eine einzige Person erfolgt, sondern durch eine zweite, von der ersten unabhängige Beurteilung überprüft wird. Diese Zweitmeinung soll von einer dafür kompetenten Person stammen.

Hinweise zur Vorbeugung von Missbrauch Neben den Empfehlungen gibt die NEK-CNE Hinweise auf weitere Bereiche, die aus ihrer Sicht eine besondere Missbrauchsgefahr beinhalten. 
In diesen besonders sensiblen Bereichen gilt es, vorbeugende Massnahmen zu treffen, um Missbräuche der straffreien Suizidbeihilfe in entsprechenden Organisationen einzuschränken.

- Es darf nicht um direkter oder indirekter finanzieller Vorteile willen gehandelt werden.

- Das Motiv zur Suizidbegleitung kann ethisch heikel sein. Ethisch nicht vertretbar sind das Ausnützen einer Notlage, die Befriedigung am Tod (Thanatophilie) oder ideologische Gründe.

- Suizidbeihilfe kann Suizidbegleiter überfordern, beispielsweise durch zu viele Begleitungen oder mangelndes Debriefing.

- Mangelnde Transparenz von Organisation und Management (inkl. Buchhaltung) einer Suizidhilfeorganisation oder mangelnde Kontrolle durch organisationsinterne und -externe Personen bzw. Sachverständige können die
Missbrauchsgefahr erhöhen. Die Gefahr besteht vor allem, wenn es sich bei der Organisation um einen nichtdemokratisch organisierten Verein mit dominanter Führungsperson oder einen Kreis handelt, der einer bestimmten Ideologie nahesteht.

\section{Literatur}

1 NEK. Beihilfe zum Suizid. Stellungnahme Nr. 9. Bern: NEK; 2005.

2 Rehmann-Sutter C, Bondolfi A, Fischer J, Leuthold M (Hrsg.). Beihilfe zum Suizid in der Schweiz. Beiträge aus Ethik, Recht und Medizin. Bern: Peter Lang; 2006.

3 SAMW. Medizinisch-ethische Richtlinien zur Betreuung von Patientinnen und Patienten am Lebensende. Basel: SAMW; 2004.

4 EJPD. Sterbehilfe und Palliativmedizin - Handlungsbedarf für den Bund? Bern: EJPD; 2006. 